\title{
Análisis estructural del comercio en Melilla
}

\author{
Marcos M. García Velasco \\ Guillermo Sarmiento Zea \\ Alejandro del Canto Bossini
}

\section{Introducción}

El comercio es una actividad casi tan antigua como la civilización humana. Surge al mismo tiempo que se empieza a desarrollar la división del trabajo.

La especialización entre las distintas tareas productivas hace necesario un intercambio de artículos, que con el tiempo dará lugar a una demanda de "intermediación". Así, además de la producción se van a realizar actividades de distribución, que como dice Dayan consisten en poner los bienes y servicios a la disposición del consumidor en las condiciones de lugar, tiempo y calidad que convienen a éste (citado por Casares Ripoll (1982), pág. 108). Es decir, es un puente entre la producción y el consumo.

El desarrollo económico y el consiguiente aumento en la especialización y concentración productiva determinada por las economias de escala va a provocar un aumento en la necesidad de intermediación con la exigencia de una mayor eficacia lo que implica bajos costes y elevados servicios.

En la evolución del sector distributivo se pueden señalar, de acuerdo con Bucklin (citado por Casares Ripoll (1981), pág. 361), las siguientes etapas: mercados, periódicos, permanentes, fragmentados e integrados verticalmente.

Los mercados periódicos se desarrollan en la época precapitalista y se establecían a intervalos, coincidiendo casi siempre con fiestas $y$ ferias de origen religioso.

La superación de la periodicidad de los mercados comienza a darse en la Edad Media, surgiendo los establecimientos con base regular de comerciantes y artesanos. Va a coincidir con la revolución de las ciudades.

El siglo XIX supone un importante punto de inflexión en la consideración de la actividad distributiva. Se produce una tendencia hacia la especialización tanto de mayoristas como detalistas hacia determinados productos, fragmentándose los mercados.

Con la crisis de 1929 se inicia una revolución en el sector distributivo con la aparición de los mercados integrados verticalmente. Esta nueva visión del comercio se va a basar en el abastecimiento directo de las fuentes de producción, intentando concentrar funciones en una sola forma comercial. 
Los fines que ha de cumplir la distribución - desde el punto de vista el consumidor- son, según Fernández Suárez (1980), los siguientes:

- La puesta a disposición de la demanda en tiempo y lugar de los bienes demandados.

- Sanidad, estabilidad y calidad del producto y del servicio.

- Una presentación adecuada (en su caso, fraccionamiento y envasado).

Ofrecer servicios ventajosos para facilitar el suministro o la adquisición.

Según Tamames (1982) la actividad comercial está integrada por las siguientes operaciones: 1) Compra y, en su caso, acopio. 2) Selección y clasificación. 3) Elaboración y empaquetado, y eventualmente, preparación para dotar al producto de nuevas cualidades. 4) Transporte. 5) Almacenaje. 6) Información de mercados y publicidad. 7) Financiación para hacer frente a los anteriores procesos. 8) Asunción de riesgos. 9) Orientación y creación de consumo. 10) Varios.

Para Casares Ripoll (1982), la distribución desarrolla una serie de funciones: material, psicológica, económica, espacial y temporal $\mathrm{y}$, por último, social.

Desde el punto de vista material, la distribución se puede concebir como el conjunto interdependiente de flujos de bienes y de medios financieros.

La función psicológica se desarrollaría a través del conjunto articulado de decisiones sobre transacciones, cantidades, calidades y precios, apoyada en flujos de información.

La distribución se configura desde el punto de vista económico, como eje estructurante y organizador de los intercambios.

Por otra parte, la distribución implica la adecuación espacial y temporal de la oferta y la demanda. El progresivo aumento de la distancia entre productor y consumidor requiere un crecimiento en la eficacia y racionalidad de la función distributiva.

En cuanto a la función social, la distribución, y más especificamente el sector comercio, se ha basado en una gran intensidad factorial del trabajo. Por lo tanto, ejerce un importante papel como generador de empleo, como sector "refugio" de la emigración rural y como estabilizador en las fluctuaciones de la economía.

Es tradicional dividir la actividad comercial en al por mayor $\mathrm{y}$ al detall siendo la primera la que facilita los artículos a los últimos y éstos quienes tratan con el consumidor directamente.

Existen múltiples clasificaciones del comercio, pero quizás la más interesante desde nuestro punto de vista sea la que recoge Gamir Casares (1980) que distingue entre sistema tradicional de distribución y sistema moderno.

De forma muy esquemática podemos decir que el sistema tradicional se caracteriza por la existencia de una actividad intensiva en trabajo y un cierto capital humano. El negocio no es intensivo ni en espacio, ni en el resto del capital humano, ni en capital físico. Se vende al consumidor no solo el producto, sino cierta información sobre el mismo, y también "se vende" una relación personal. Enfrente hay un consumidor de baja renta, baja movilidad en sus desplazamientos, con un hogar no preparado para el almacenamiento de productos perecederos, baja cultura, etc.

La aparición y posterior desarrollo del automóvil y el aumento de movilidad 
que ello implica, la adquisición de información sobre la mercancia (fundamentalmente a través de las campañas publicitarias) sin necesidad del comerciante y la expansión de utilización de frigorificos y congeladores, junto con la fuerte elevación del precio relativo del factor trabajo va a provocar, como dice Gámir Casares, una crisis del sistema tradicional de distribución.

Los nuevos sistemas implican una actitud del consumidor cada vez más activa, una sustitución de trabajo por espacio, lo que supondrá algunas inversiones, y que exigirán un capital humano distinto al del comercio tradicional. Como dice Fernández Suárez, la distribución moderna va à vender unidades de servicios más que mercancias.

La principal ventaja para el consumidor derivada de los nuevos sistemas se va a reflejar en el precio del artículo.

Pero como dice el propio Gámir Casares, la crisis del sistema tradicional de distribución y la aparición de nuevos sistemas no implica la desaparición del "pequeño comercio", sino su transformación.

Las principales tendencias del comercio minorista son recogidas por Casares Ripoll (1983), quien distingue entre establecimientos de pequeña o mediana dimensión y los de grandes superficies.

Entre los de pequeña o mediana dimensión se encuentran los establecimientos de proximidad, los de descuento, los superservicios y supermercados y las tiendas especializadas.

Dentro de las grandes superficies cabe hablar de los centros comerciales, distinguiéndose dentro de los mismos entre los de almacenes especializados por secciones y departamentos, y los almacenes de consumidor.

Las caracteristicas de las distintas clases de establecimientos mencionados pueden verse en Casares Ripoll (1983), por lo que aquí no nos vamos a extender en ello.

El comercio en Melilla

\section{1.-Su importancia}

El comercio tiene una gran importancia en Melilla, y ello se puede ver observando que en 1981, según datos del Censo de Población, una de cada cinco personas ocupadas, lo estaba en este sector; mientras que para el conjunto nacional la relación era de uno a diez aproximadamente. Junto con el sector de Administración Pública, Defensa y Seguridad Social, mantiene cerca de la mitad de la población ocupada, con lo que se configura como uno de los pilares más importantes que sustentan la economía de Melilla, cuyo estudio se hace por tanto indispensable para poder determinar la estructura económica de la ciudad. 


\section{2.-Consideraciones metodológicas}

El presente trabajo trata de analizar las características de la estructura comercial de la ciudad de Melilla. Para ello, este estudio ha sido dividido en varias partes.

En la primera se estudian las características físicas de los locales y establecimientos comerciales, es decir, el capital real o material sobre el que se basa esta actividad. A continuación se analiza el elemento personal o humano desde una doble perspectiva: la población ocupada y el empresario. Por último, se va a estudiar la actividad comercial en su funcionamiento. Finalizaremos el trabajo considerando el grado de implantación de los nuevos sistemas de venta y las tendencias del comercio en nuestra ciudad.

Pero antes de entrar en materia conviene hacer una matización. En un primer acercamiento al universo comercial hemos observado que no se puede realizar una clara y tajante división entre mayoristas y minoristas, pues en multitud de casos ambos tipos de actividades se realizan conjuntamente.

Cuando examinamos el comercio mayorista se observa una gran diversidad de situaciones particulares existentes. Esta hetereogeneidad hizo necesario aplicar criterios restrictivos en su análisis pues no todos los empresarios con licencia comercial de mayorista pueden ser considerados como tales, en un criterio más ajustado de distribución comercial.

Se puede constatar que el número efectivo de comerciantes mayoristas, en sentido estricto, es significativamente inferior que las cifras obtenidas por licencias comerciales.

Por otro lado, un número importante de minoristas realizan conjuntamente actividades mayoristas, debido al habitat en que se desenvuelven, y a que la especificación de sus productos es elevada.

Además de lo anterior hay que decir que muchas de las características del comercio minorista se pueden aplicar al mayorista, y viceversa. Por tanto, salvo excepciones, nuestro trabajo no va a distinguir entre estos dos tipos, sino que se va a realizar considerando, en la mayoría de los casos, ambas actividades conjuntamente.

\section{3. - Las estructuras comerciales}

\section{1.-El factor fisico}

\subsection{1.-Localización fisica del establecimiento}

Una cuestión muy importante cuando se plantea la instalación de una industria o negocio es la de determinar su localización, siendo múltiples las teorias elaboradas al respecto. Por tanto, este trabajo va a comenzar analizando cómo se distribuyen en el espacio los establecimientos comerciales. 
La mayor parte de los establecimientos están concentrados en el centro de la ciudad y principalmente en un área delimitada por el Parque Hernández y la calle Pablo Vallescá. Habría que destacar la agrupación de bazares en el sector denominado Mantelete. También hay que mencionar otra zona de un importante movimiento comercial y que se localiza en las cercanias del mercado central y la calle General Margallo.

La mayor concentración se da en el sector de bazar y en el alimenticio que se agrupa en torno a los mercados de abastos. La máxima presencia la da frutas y verduras, pescados y mariscos y carnicerias y charcuterias, como es lógico por otro lado. Sin embargo, la mayoria de los establecimientos minoristas tienen una localización aislada de los demás comercios.

\subsection{2.-Dimensión}

Otro tema básico en el estudio de una empresa es la dimensión, pues dependiendo de la misma se pueden establecer las necesarias economias de escala que disminuyan los costes y permitan reducir los precios pagados por los consumidores, aumentando la producción y el consumo.

La dimensión de la empresa puede ser estudiada desde distintos puntos de vista. Aquí sólo vamos a considerar los dos aspectos que suelen ser más analizados en la actividad comercial: el número de personas ocupadas por establecimiento y la superficie de venta.

Según el Censo de Locales, en 1980 en Melilla había 1.268 establecimientos, con 2.595 personas ocupadas en los mismos, lo que hace una media aproximada de 2 personas por establecimiento.

Dentro del comercio al por menor los subgrupos de menor dimensión son: frutas y verduras, huevos y aceite, carnicería y charcuteria y pescados y mariscos; es decir, aquellos comercios especializados de alimentación. No obstante, todo el grupo de alimentación tiene una relación persona ocupada-establecimiento bastante baja $(1,4)$.

En el sector mayorista tienen una baja relación el de textiles, y confección y calzado, con 1,5 personas por establecimiento. Esto contrasta con el valor correspondiente a este mismo grupo del comercio minorista que es superior a esa cifra.

Las mayores relaciones persona-establecimiento, se dan en el comercio al por mayor de productos farmacéuticos, y en el comercio minorista de automóviles.

El 42,3 por ciento de las personas ocupadas en el comercio al por mayor lo estaba en locales-establecimientos de más de 5 personas, no existiendo ninguno con una cifra superior a 50. Sólo 2 establecimientos correspondientes a materiales de construcción tenían más de 20 personas.

En cuanto al comercio minorista, sólo el 17,3 por ciento de las personas ocupadas lo estaban en locales-establecimientos de más de 5 , no existiendo al igual que en el comercio al por mayor ninguno con más de 50 .

Solo en el subsector de automóviles más del 50 por ciento de las personas ocu- 
padas lo estaban en establecimientos de más de 5 personas, mientras que alimentación, productos farmacéuticos, droguería y perfumería lo estaba en su casi totalidad en los de menos de 5.

Referente al espacio físico observamos que el tamaño de los establecimientos minoristas dedicado a la venta es muy reducido, existiendo un número muy elevado de comercios con una superficie igual o inferior a 40 metros cuadrados y siendo la mayoría inferior a 60 . No existe ninguno con una extensión superior a 400 (1). Por otra parte, la media de la superficie de los establecimientos mayoristas suele situarse en torno a los 200 metros cuadrados.

Todo lo anterior nos indica que los establecimientos comerciales de Melilla son de pequeña dimensión, lo que es lógico, por otro lado, dado el tamaño del mercado local.

\subsection{3.-Características de los locales}

Como ya hemos señalado, el espacio físico donde se localiza la actividad comercial es un factor importante para evaluar las características del comercio y las posibilidades de reforma de las estructuras.

Como dice el IRESCO (1983): "No ofrece las mismas posibilidades un local en régimen de alquiler que otro en propiedad; no es igual transformar locales diseñados para los comercios de hace dos o tres décadas, donde básicamente la mediana y gran superficie apenas existía, que intentar incorporar en dichos espacios fisicos las características y tendencias que presenta el comercio de nuestros días."

Con relación a lo anterior hemos analizado dos variables: el régimen de tenencia de los establecimientos y la antigüedad de los inmuebles.

Podemos decir que del 90 al 95 por ciento de los locales son alquilados y además tienen una elevada antigüedad. Esto es evidente puesto que la mayoría de los edificios de Melilla datan de primeros de siglo y la construcción en nuestra ciudad ha sido muy escasa durante bastante tiempo. Constituyen una excepción a lo anteriormente dicho las galerías comerciales del edificio Anfora y del antiguo cine Monumental.

El predominio del régimen de alquiler se debe principalmente a que los propietarios de los locales no están interesados en vender puesto que la escasa actividad en el sector de la construcción en nuestra ciudad ha provocado que la demanda exceda a la oferta y por tanto se den elevados traspasos y rentas. Aparte de la falta de consideración en la redacción de los planes de ordenación urbana de las necesarias reservas de suelo para las actividades comerciales.

(1) A partir de 400 metros tenemos los supermercados, hipermercados, grandes almacenes, y otros tipos de establecimientos de grandes superficies. 


\section{2.-El factor humano}

\subsection{1.-La población ocupada en el comercio: su distribución por sexo}

La primera característica que se aprecia al observar la distribución por sexo del comercio en Melilla es la gran dedicación de los varones, ya que en 1981 según el Censo de Población el 23,6 por ciento del total de la ocupación masculina lo estaba en este sector, mientras que en las mujeres este porcentaje era del 16,6 por ciento. Por el contrario, para el conjunto nacional la relación del sexo femenino es muy similar a la de Melilla, mientras que para los hombres es mucho más baja (2).

La participación tanto de varones como de mujeres en el comercio va a crecer entre 1970 y 1981, lo que muestra una tendencia hacia una mayor especialización de la economía de Melilla en este sector, pero el aumento en el caso del sexo masculino es mayor al experimentado por el otro (3).

En cuanto a la edad, la primera característica a destacar es que el comercio emplea a más del 40 por ciento de la población ocupada menor de 20 años, lo que nos muestra la gran importancia que tiene este sector dentro de la economía de Melilla en los estratos de edad más baja (además, el 40 por ciento de la población ocupada de esta edad tenía en 1981 la profesión de dependiente de comercio). Si por otra parte observamos que más del 30 por ciento de los ocupantes mayores de 65 años eran comerciantes, vemos que la influencia es aún muy superior en los estratos jóvenes y de edad más avanzada.

Si a lo anterior se añade que más de las tres cuartas partes de los responsables de establecimientos comerciales (directores, gerentes y propietarios), tenían en 1981 más de 40 años, podemos deducir que la estructura predominante en las relaciones laborales se acerca más a patrones decimonónicos que los que se dan en la actualidad.

\subsection{2. - La distribución por sectores}

Según el Censo de Locales de 1980 destaca por su importancia el grupo de comercio al por menor de productos alimenticios, que suponía el 42 por ciento del total de establecimientos detallistas y el 33 por ciento de las personas ocupadas en ellos.

Dentro de este grupo tiene un alto predominio el de alimentación general, que supone más del 60 por ciento de la actividad, mientras que los establecimientos especializados no llegan al 35 por ciento. Para el conjunto nacional, según el

(2) En el conjunto nacional el porcentaje de ocupación en el comercio de la población masculina era del 9 por ciento, mientras que la tasa femenina era del 13 por ciento.

(3) En 1970, las relaciones porcentuales de ocupación en el comercio de varones y mujeres eran de 20,9 y 15,1 respectivamente. 
IRESCO (1981), los porcentajes respectivos son del 40 y 56 por ciento aproximadamente. Como se ve, en Melilla la especialización en este tipo de comercio es muy baja.

Si observamos el resto de los sectores se puede apreciar que también se da una baja especialización en los establecimientos comerciales.

Otra caracteristica de la distribución del comercio por sectores en Melilla es la importancia que tienen "electrodomésticos y menaje", y "otros comercios no considerados" (4), junto con "carburantes y lubricantes", en relación con el conjunto nacional. Todos estos subgrupos suponen aproximadamente el 20 por ciento del total de establecimientos y personas ocupadas en el comercio minorista.

Hay que destacar la inexistencia en el Censo de Locales de establecimientos organizados por secciones, como supermercados, hipermercados, grandes almacenes, etc...

\subsection{3.-Caracteristicas del empresario}

La importancia del empresario individual es primordial ya que como veremos la mayoría de los comercios tienen como forma jurídica predominante la de "comerciante individual", y en ellos, la figura de la persona que administra directamente su negocio es preponderante.

La edad media del comerciante individual en Melilla es de 46 años. Sin embargo, las tres cuartas partes de los empresarios tienen más de 40 años (5), lo que supone un grado de envejecimiento muy importante dentro del sector. A pesar de todo la media es muy similar a la nacional (6). En cuanto al nivel de formación de los empresarios podemos señalar que es bastante bajo, puesto que la gran mayoría sólo tienen estudios primarios. Sin embargo hay que destacar el hecho de que desde hace algunos años se observa que muchos hijos de comerciantes que han cursado estudios medios y superiores están integrándose en los negocios familiares. Hecho que consideramos de una relevante importancia, puesto que gran parte de las reformas estructurales que está necesitando nuestro sistema distributivo, pueden venir dadas por esta nueva generación de empresarios.

Asimismo, estimamos que seria conveniente el incremento en la realización de cursos y seminarios por parte de organismos estatales y empresariales, que impartiesen las enseñanzas necesarias para que este sector alcance el grado de modernización que requiere (7).

(4) En estas dos categorias se pueden incluir los bazares.

(5) Datos obtenidos según el Censo de Población de 1981.

(6) La edad media del empresario nacional es de 47 años (IRESCO, 1983).

(7) Entre los organismos que imparten cursos hay que destacar al IRESCO y al IMPI (Instituto para el desarrollo de la pequeña y mediana empresa). Este último realizó el año pasado un curso sobre comercio exterior en la Cámara Oficial de Comercio de nuestra ciudad. 


\section{3.-Caracteristicas funcionales}

\subsection{1.-Técnicas de venta}

Prácticamente la totalidad de los establecimientos comerciales utilizan técnicas tradicionales, es decir, la venta a través del mostrador. Sólo en muy pocos dedicados a la alimentación existe el régimen de autoservicio, pero incluso en este sector, que es quizás el más indicado para la utilización de este procedimiento, predomina aún la clásica tienda y no existen supermercados, hipermercados, ni grandes almacenes. También consideramos importante destacar que se dan numerosos establecimientos de reducida superficie en los que se puede adquirir una gran variedad de artículos muy diversos.

\subsection{2.-Tipo de clientes y márgenes brutos}

Dentro de este apartado hay que decir en primer lugar que las ventas de mayoristas no tienen por principal destinatario al comercio al por menor de nuestra ciudad, sino que una parte muy importante de su clientela está constituida por residentes en el extranjero. Del total de las mercancías facturadas se estima que entre un 60 y un 90 por ciento no tiene como destino el mercado local.

El margen bruto con que suele trabajar el comerciante medio lo podemos estimar en un 10 por ciento sin embargo este dato hay que considerarlo con la máxima reserva, pues además de la dificultad de obtener cifras fiables hay que contemplar el artículo y la existencia o no en plaza del mismo.

Estos márgenes tan reducidos, que llegan incluso a ser menores, se deben a que determinados clientes realizan un gran volumen de compras con pago al contado.

En el caso del comercio al por menor existe una gran amplitud en los márgenes, dependiendo del sector y del cliente.

\subsection{3.-Sistemas de aprovisionamiento y formas de pago}

La política de compras es un factor esencial para la eficacia de la empresa y de ella depende en gran medida la rentabilidad del negocio. Los sistemas de aprovisionamiento empleados son muy variados dependiendo del tipo de empresa.

Antes de pasar revista a las políticas de compras por sectores, conviene decir que en los casos de droguería y perfumería, textiles y cuero, y artículos de equipamiento del hogar, no se puede establecer una ciara división entre establecimientos mayoristas y de venta al detall.

-Alimentación y bebidas. Los mayoristas se suelen proveer directamente de fábrica y de importaciones (principalmente de la CEE). En cuanto a los minoristas, éstos usualmente compran a los primeros. 
Hay que destacar la existencia de algunos establecimientos que realizan simultáneamente la venta al por mayor y al detall.

-Droguería, perfumería y productos farmacéuticos. En droguería y perfumería las compras suelen realizarse directamente a fábrica $o$ a través de representantes.

En el caso de los productos farmacéuticos, los minoristas se surten a través de mayoristas. Estos últimos compran directamente a los laboratorios o por intermedio de representantes. .

-Textiles y cueros. Los pedidos se realizan normalmente a fábrica, a través de representantes. También se dan importaciones directas, sobre todo de Extremo Oriente (Taiwan, Hong Kong, etc.) y Marruecos (principalmente pieles y curtidos).

- Artículos de equipamiento del hogar. Los principales proveedores de este tipo de artículos son los fabricantes, importadores, o a través de representantes.

Una característica muy importante es la práctica inexistencia de devolución de mercancía a los proveedores establecidos fuera de plaza, debido al aislamiento geográfico y problemas de orden administrativo y fiscal. Las dificultades de transporte junto con las derivadas del paso de aduana, devolución de impuestos, etc., obliga a los comerciantes a realizar sus compras en firme, lo que lógicamente va a incidir en el precio final del artículo.

El optimizar los sistemas de abastecimiento es uno de los objetivos prioritarios para la buena gestión económica de la empresa. Tanto en el comercio al por menor como al por mayor así como entre ellos puede ser interesante la agrupación de comerciantes con el fin de efectuar las compras conjuntamente beneficiarse de las ventajas que lleva aparejada la adquisición de un volumen elevado de mercancias.

En nuestro caso podemos decir que se está muy lejos de conseguir lo que podriamos denominar, una auténtica articulación del sector comercial puesto que no se han implantado cadenas sucursalistas, cooperativas, de minoristas ni agrupaciones de compras, tampoco existe la manipulación y transformación de los productos.

Dentro de la política de aprovisionamiento es de resaltar la gran incidencia dentro del sector mayorista de las relaciones comerciales directas con proveedores extranjeros, destacando las empresas dedicadas a la comercialización de bienes de consumo duradero. Podemos poner de manifiesto el importante comercio existente entre mayoristas, porque unos utilizan a otros como proveedores, siendo la actividad de alimentación la que fundamentalmente utiliza esta práctica, de forma que este escalón de la distribución se desdobla en dos o mas para ejercer la función de mayorista.

Las formas de pago predominantes en nuestra ciudad son las de contado junto con operaciones documentadas de plazo no superior a 90 días. De esta forma, los créditos superiores a tres meses son casi inexistentes. Si a esto se une lo anteriormente dicho sobre devoluciones de mercancías no podemos más que concluir que nuestro comercio tiene una alta dependencia de los proveedores. 


\subsection{4. - Forma juridica predominante}

La mayoría de los establecimientos comerciales adoptan la forma juridica de comerciante individual, sin apenas existir sociedades.

Sin embargo, en el comercio mayorista se observa que algunas empresas adoptan la forma juridica de sociedad.

\subsection{5.- Nivel de automatización en la gestión}

En este apartado nos referiremos a los sistemas de información más utilizados, a los datos que tienen mayor importancia para valorar la gestión económica y al grado de automatización con que se recogen y procesan esos datos.

Los procedimientos usuales de información son los tradicionales y apenas existen sistemas informatizados. Generalmente sólo se lleva una gestión detallada de clientes y proveedores. También hemos de añadir que existe un alto porcentaje de comercios que utilizan los servicios de asesorias, pero su objetivo suele ser de tipo fiscal y no de orden económico. Se observa que la informática se está introduciendo, aunque tímidamente, en el sector mayorista.

Hoy dia y como consecuencia de los avances realizados en el campo informático, entre los cuales destacariamos la disminución de precios, la miniaturización de los componentes electrónicos con la aparición de los microprocesadores y los VLSI (8), el empresario dispone de una herramienta que le permite administrar y gestionar su negocio con una mayor eficiencia y a un coste muy asequible.

Por tanto sería deseable la sustitución de los métodos tradicionales de gestión por otros más informatizados y en definitiva más eficaces.

\section{4.-Tendencias del comercio mayorista}

El comercio mayorista no constituye un bloque homogéneo y es fácil encontrar en él diferencias significativas entre una y otras actividades, $y$ aún dentro de las mismas entre una y otra empresa.

En este sentido, se constata que un buen número de empresas han iniciado una tímida evolución en los últimos años hacia una modernización de la distribución. Así mismo existe otro amplio grupo de empresas con estructuras inadecuadas en cuanto a dimensión, equipamiento, etc...

Podemos afirmar que si bien ha acontecido una transformación en el comercio mayorista no lo ha sido de una manera uniforme y en todas las empresas. El cambio solo se ha producido en alguna de ellas de diferente forma y medida. Los sectores que han estado más ligados a la sociedad de consumo son los que más se han

(8) Circuitos de nivel muy elevado de integración. 
expandido y los que presentan una configuración más moderna, de cualquier forma tenemos que resaltar el bajo nivel que registra este factor en el desarrollo de técnicas de almacenamiento y bienes de equipos utilizados en los establecimientos.

\section{5.-Los nuevos sistemas de comercio}

En cuanto a los nuevos sistemas de comercio, en nuestra ciudad los establecimientos de este tipo son escasos ya que sólo son siete de los que cuatro han sido calificados como autoservicios y tres superservicios (9).

Ninguno de ellos tiene una superficie mayor de 200 metros cuadrados, ni más de cuatro personas asalariadas, realizando la apertura al público con fecha posterior a 1980 la mayoria de ellos.

\section{6.-Conclusiones}

La primera conclusión que debemos resaltar es la gran importancia que tiene el comercio en nuestra ciudad; ello se refleja en la gran proporción de mano de obra masculina dedicada a esta actividad.

El patrón de relaciones laborales que se suele dar es el tradicional, en el que se accede a muy temprana edad sin apenas cualificación desarrollándose la formación a lo largo del tiempo dentro de la misma empresa.

Otra caracteristica muy especifica del comercio en Melilla es la gran importancia relativa que tienen los bazares, que le otorgan una especial fisonomia y configuración.

Además, el comercio se caracteriza por estar poco especializado y ofrecer al mismo tiempo una gran diversidad de artículos.

El funcionamiento del comercio en Melilla es muy peculiar. La mayor parte de las ventas no se destinan al mercado local, realizándose éstas, en la mayoría de los casos, con bajos márgenes de beneficio, debido a que estas operaciones son al contado y por un gran volumen de mercancías.

Asimismo, no podemos dejar de destacar la gran dependencia que se tiene de los suministradores exteriores. Ello se debe al aislamiento geográfico de nuestra ciudad, aparte de problemas administrativos y fiscales. Todo esto se traduce en que en multitud de ocasiones hay carencia de determinados artículos, siendo una expresión muy común la de "de este artículo no hay en plaza".

Para finalizar, solo nos resta reiterar la gran importancia que tiene el comercio en nuestra ciudad, caracterizándose éste por unas estructuras que se pueden calificar como tradicionales. Sería deseable por tanto una modernización de este sector intentando disminuir su dependencia del exterior.

(9) Calificación otorgada por la Dirección General de Comercio Interior el 28-09-83. 
Aparte, opinamos que uno de los objetivos prioritarios sería el de buscar una mayor diversificación de la estructura económica, fomentando otros sectores distintos.

\section{REFERENCIAS BIBLIOGRAFICAS}

CASARES RIPOLL, Javier (1981): "La politica de reforma estructural del comercio interior mediante la ayuda a la pequeña y mediana empresa comercial" Lecturas de Economia Española e Internacional. SGT del Ministerio de Economía y Comercio. Madrid, 1981.

- (1982): "Dualismo en el comercio interior." Información Comercial Española. Núm. 582, febrero, 1982, pág. 108-110.

- (1983): Los cambios en el "giro de la rueda". Tendencias en el comercio minorista. ICE. Nüm.603, noviembre, 1983 , págs. $68,75$.

FERNANDEZ SUAREZ, A. (1980): "Visión general de la politica de comercio interior". Política Económica de España. Coord. por L. Gámir Casares. Alianza Universidad. Madrid, 1980.

GAMIR CASARES, L. (1980): "Política de transformación de las estructuras comerciales interiores." Política Económica de España. Coord. por L. Gámir Casares. Alianza Universal. Madrid, 1980.

IRESCO (1983): "Caracteristicas Estructurales del comercio en España”. IRESCO, Ministerio de Economía y Hacienda. Madrid, 1983.

TAMAMES, R. (1982): "Estructura económica de España". Alianza Universidad. Madrid, 1982. 